\title{
Correlative Enhanced Scattering in the upper hybrid resonance for study of micro and meso-scale wave phenomena in low-temperature and tokamak plasmas
}

\author{
Evgeniy Gusakov ${ }^{1}$ \\ Ioffe Institute \\ 26 Politekhnicheskaya, St. Petersburg, Russia \\ E-mail: evgeniy.gusakov@mail.ioffe.ru
}

\author{
Alexey Gurchenko \\ Ioffe Institute \\ 26 Politekhnicheskaya, St. Petersburg, Russia \\ E-mail: aleksey.gurchenko@mail.ioffe.ru
}

\begin{abstract}
The paper presents results of development of microwave correlative enhanced scattering (CES) diagnostics performed during the last decade. The CES technique is a backscattering diagnostic utilizing simultaneous $X$-mode plasma probing at different frequencies in the presence of the upper hybrid resonance (UHR). The diagnostic is benefiting from the growth of electric field and wave numbers in the UHR resulting in enhancement of scattering signal, sub-mm resolution and possibility to measure locally the wave number and frequency spectra of turbulent fluctuations. The high performance of diagnostics has been demonstrated both in laboratory low-temperature plasma and in tokamak experiments. In this paper after the CES introducing results of its application to study of the following physical phenomena are presented:
\end{abstract}

1. The first observations of the small-scale ETG-mode drift wave turbulence performed in FT-2 research tokamak.

2. Exponential TEM-mode radial wave number spectra in tokamak.

3. Enhanced Doppler effect in the UHR backscattering and investigation of tokamak geodesic acoustic mode (GAM) spatial structure using CES.

4. Benchmarking of the global full-f gyrokinetic computations against CES data.

5. Anomalous RF power absorption mechanism investigation in low-pressure helicon discharge performed by CES technique which resulted in visualization of ion acoustic parametrically driven turbulence.

First EPs Conference on Plasma Diagnostics - $1^{\text {st }}$ ECPD

14-17 April 2015,

Villa Mondragone , Frascati (Rome) Italy

${ }^{1}$ Speaker 


\section{Introduction}

Enhanced microwave scattering (ES) is a powerful method for diagnosing small-scale fluctuations or waves in magnetised plasma [1]. This diagnostics makes use of the extraordinary wave for probing, and backscattering (BS) off low-frequency (LF) electron density fluctuations in the Upper Hybrid Resonance (UHR) determined by condition $\omega_{i}^{2}=\omega_{p e}^{2}(x)+\omega_{c e}^{2}(x)$ is detected by it. When the incident wave of frequency $\omega_{i}$ approaches the resonance point, the wave number grows strongly up to values much larger than the vacuum value $k_{i}=\omega_{i} / c$. Along with the wave number, the amplitudes of the incident and BS waves increase, thus resulting in strongly enhanced scattering signals. Furthermore, the spatial resolution is high since this process is localised to the resonance region. The method also permits to measure the spatial distribution of the fluctuations by varying the probing frequency thus displacing the UHR layer.

In toroidal devices, where the UHR and magnetic surfaces do not coincide due to dependence of magnetic field on the major radius $R$, the large probing wave number $k_{r}$, perpendicular to the UHR surface, has a finite projection onto the poloidal direction [2]. In general case the probing wave vector poloidal projection $k_{\theta}$ is given by relation

$$
k_{\theta}=k_{\theta 0}-\left.k_{r} \frac{\vec{e}_{\theta} \vec{e}_{R} \omega_{\mathrm{ce}}^{2}}{R\left|\vec{\nabla}\left(\omega_{\mathrm{pe}}^{2}+\omega_{\mathrm{ce}}^{2}\right)\right|}\right|_{\mathrm{UHR}},
$$

where $k_{\theta 0}$ is the probing $X$-mode poloidal wave number out of the UHR zone, $\vec{e}_{\theta}$ and $\vec{e}_{R}$ are unit vectors in poloidal and major radius directions. Due to large radial component of the wave vector the poloidal projection given by (1) could substantially exceed the vacuum value thus leading to growth of the Doppler frequency shift at backscattering (Enhanced Doppler effect).

Since the incident wave number strongly changes near the UHR, the Bragg condition for backscattering can be fulfilled in a wide range of fluctuation scales contributing to the BS signal. Due to this integral feature, less a priori information on the fluctuation properties is generally needed for designing the scattering diagnostics.

One of the methods to achieve the wave number resolution in the ES diagnostics is based on application of the time-of-flight technique [2-4]. It makes use of the fact that the time delay of the ES signal depends linearly on the wave number of fluctuations and utilizes the RADAR or frequency sweep method. Although the time-of-flight ES technique enables one to determine the wave number spectra of small-scale plasma fluctuations, it has drawbacks and limitations. Namely, in the RADAR method, the signal-to-noise ratio is small as compared to the stationary ES technique while the sweep technique permits merely investigation of narrow frequency band plasma wave spectra and, thus, cannot be applied to study turbulence.

Another modification, namely the Correlative ES (CES) diagnostics, that combines the virtues of the stationary method (high sensitivity) and the time-of-flight techniques (wave number resolution) is based on the dependence of the ES signal on the phase of the fluctuations in the BS point. It was first proposed in [5], justified theoretically in [6] and then applied to investigation of turbulent phenomena in tokamak and laboratory plasma.

\section{Theoretical background}

To be illustrative of the main features of the CES, we calculate the phase of BS wave assuming slab geometry for the scattering, which is a reasonable approximation in the vicinity 
of the UHR. Furthermore, we assume that the WKB approximation holds for the probing waves as well as the scattered waves. Under the above assumptions, the phase of the backscattered wave $\Phi_{s}$, which is determined by the phases of the incident wave $\Phi_{i}$ and a fluctuation $-q x_{s}$ in the scattering point and the phase variation of the scattered wave on the way back to the antenna, is given by

$$
\Phi_{s}=\int_{x_{a}}^{x_{s}} k_{i}\left(x^{\prime}\right) d x^{\prime}-q x_{s}+\int_{x_{a}}^{x_{s}} k_{s} d x^{\prime}=2 \int_{x_{a}}^{x_{s}} k_{i}\left(x^{\prime}\right) d x^{\prime}-q x_{s},
$$

where $x_{a}$ is the antenna position and the BS point $x_{s}$ is determined by the Bragg condition

$$
k_{i}(x)-k_{s}(x)=2 k_{i}(x)=q .
$$

We neglect here the weak spatial variation of the fluctuation wave number compared to the steep growth of it for the UH waves. In the case of scattering in the inhomogeneous medium the BS point determines the centrum of volume contributing to the scattering signal. The width of this volume $l$, which determines the spatial localisation of the UHR scattering experiment in the inhomogeneity direction is given in the WKB approximation by $l=\left|2 d k_{i} / d x\right|_{x=x_{s}}^{-1}$. From condition (3), we obtain the frequency dependence of the scattered phase as

$$
\frac{\partial \Phi_{s}}{\partial \omega_{i}}=2 \int_{x_{a}}^{x_{s}} \frac{\partial k_{i}}{\partial \omega_{i}} d x^{\prime}
$$

This is equal to the time delay of the BS signal, which is in the vicinity of the UHR [3] given by

$$
t_{d}=-q \frac{\partial x_{U H}}{\partial \omega_{i}}+t_{0} .
$$

The second term $t_{0}$ associated with the propagation far from the UHR is generally small as compared to the first term. The phase of the ES signal can then be expressed by

$$
\Phi_{s}=\int^{\omega_{i}} t_{d} d \omega^{\prime}=-q x_{U H}\left(\omega_{i}\right)+\Theta
$$

where the first term on the right-hand side varies strongly with $\omega_{i}$ due to the large fluctuation wave number $\left(q>>=\omega_{i} / c\right)$, whereas the second term, that is related to the propagation in the plasma far from the UHR, is usually small. The phase of the ES signal originating from the propagation in the microwave circuit is omitted in the above expression and should be compensated in the experiment. The fact that the phase of the ES signal is determined by the phase of the fluctuation in the UHR may be used to measure the fluctuation wave number and the turbulence wave number spectrum by applying the correlation technique. The crosscorrelation function of ES signals at probing frequencies $\omega_{1}$ and $\omega_{2}$, according to [6], is given in terms of distance between the UHR points $\Delta x_{U H}=x_{U H}\left(\omega_{1}\right)-x_{U H}\left(\omega_{2}\right)$ as

$$
K\left(\Omega, \omega_{1}, \omega_{2}\right)=\left(\frac{\omega}{4 \pi n_{e}}\right)^{2} \int\left[|U(\vec{q})|^{2}+|U(-\vec{q})|^{2}\right]\left|\delta n_{\vec{q}, \Omega}\right|^{2} e^{-i q_{x} \Delta x_{U H}} \frac{d^{3} \vec{q}}{(2 \pi)^{3}},
$$

which is suitable to determine the cross-power wave number spectral density

$$
I_{q_{x}, \Omega}=\int\left[|U(\vec{q})|^{2}+|U(-\vec{q})|^{2}\right]\left|\delta n_{\vec{q}, \Omega}\right|^{2} \frac{d q_{y} d q_{z}}{(2 \pi)^{2}} .
$$

The expression for it is provided by the Fourier transform of (7) in the form 


$$
I_{q_{x}, \Omega}=\frac{\left(4 \pi n_{e}\right)^{2}}{\omega^{2}} \int_{-\infty}^{+\infty} e^{i q_{x} \Delta x_{U H}} K\left(\Omega, \Delta x_{U H}\right) d\left(\Delta x_{U H}\right)
$$

where we supposed $\omega_{1} \approx \omega_{2}=\omega$. The form-factor $|U(\vec{q})|^{2}$ is proportional to the scattering efficiency that depends on the wave number $q_{x}:|U(\vec{q})|^{2}=S\left(q_{x}\right)\left|F\left(q_{y}, q_{z}\right)\right|^{2}[1]$. The factor $\left|F\left(q_{y}, q_{z}\right)\right|^{2}$ here is only determined by the antenna diagram. For tokamak plasma at $2 k_{c}<q_{x}<<2 \min \left\{\left(l l_{T}^{2}\right)^{-1 / 3}, k_{c}^{2} l\right\}$ the $S\left(q_{x}\right)$ dependence is cubic $S\left(q_{x}\right) \sim q^{3}$. For higher wave numbers it became oscillatory, as it is shown in Fig. 1(a) for the FT-2 tokamak experimental parameters. For small-sized plasmas and large probing wavelengths, when the condition $k_{c} l<<1$ holds, the scattering efficiency is given by expression $S\left(q_{x}\right)=q_{x}^{2} e^{-2 q_{x} l \varepsilon^{i m}}$ which is valid for $0<q_{x}<<2\left(l l_{T}^{2}\right)^{-1 / 3}$ (here $\varepsilon^{i m}$ is the imaginary part of the dielectric tensor component).

The density perturbation spectrum, weighted with the antenna diagram

$$
\bar{N}^{2}\left(q_{x}, \Omega\right)=\int|F|^{2}\left|\delta n_{\bar{q}, \Omega}\right|^{2} \frac{d q_{y} d q_{z}}{(2 \pi)^{2}}
$$

is expressed as

$$
\bar{N}^{2}\left(q_{x}, \Omega\right)=\frac{I_{q_{x}, \Omega}}{S\left(q_{x}\right)}
$$

It should be mentioned that this spectrum, obtained from the experimental data through (11) is complex whereas it must be real according to (10). The imaginary part of $\bar{N}^{2}\left(q_{x}\right)$ can be used to estimate the accuracy of the correlation analysis.

\section{Experimental ES applications in tokamak}

\subsection{Small-scale TEM and ETG mode investigation}

The first CES diagnostic application at the tokamak was focused on the search for the ETG mode. The experiments were performed at the FT- 2 tokamak $\left(R=55 \mathrm{~cm}, a=8 \mathrm{~cm}, B_{\mathrm{T}} \approx 2.2 \mathrm{~T}\right.$, $\left.I_{\mathrm{p}}=19 \div 37 \mathrm{kA}, \quad n_{\mathrm{e}}(0)=(2 \div 4) \times 10^{19} \mathrm{~m}^{-3}, \quad T_{\mathrm{e}}(0) \approx 500 \mathrm{eV}\right)$ where a movable focusing double antennae set, allowing off equatorial plane plasma $X$-mode probing from high magnetic field side with maximal vertical displacement up to $y_{\mathrm{a}}= \pm 2 \mathrm{~cm}$ is installed. The probing wave is launched into the plasma perpendicular to the magnetic field under conditions when the electron cyclotron resonance layer exists in the chamber and thus the UHR is accessible. The beam radius at the UHR position, for the probing frequencies $\left(f_{\mathrm{i}}=52-69 \mathrm{GHz}\right)$ is $\rho=0.7 \div 0.9 \mathrm{~cm}$. The BS wave is received by the second antenna of the double antenna set. The CES experiment geometry is shown in Fig. 1(b). The probing wave UHR layer was situated at $r>4 \mathrm{~cm}$.

Two types of backscattering spectra were measured in ohmic discharges. The first one, shown in Fig. 1(c) by curve 1, is observed in the plasma edge at $7.9 \mathrm{~cm}>r>6 \mathrm{~cm}$. It is characterized by high level, symmetrical form and smaller Doppler frequency shift of 0.2 $0.5 \mathrm{MHz}$ corresponding to the electron diamagnetic drift direction. The second, shown in Fig. 1(c) by curve 2, is usually observed in the internal plasma region at $6 \mathrm{~cm}>r>4 \mathrm{~cm}$, where condition for the ETG mode excitation [2] $L_{T}<1.25 L_{n}$ was fulfilled. It possesses huge 
frequency shift of $1.5-3 \mathrm{MHz}$ and smaller level. The backscattering satellite in this case is always broader and less symmetric. In many cases it is composed of two satellites, as shown in Fig. 1(c). The amplitude of the low frequency (LF) satellite decreases when moving inward the plasma whereas the high frequency (HF) satellite's amplitude increases [8]. The observation of a double line in the UHR BS signal is most likely associated with simultaneous excitation of two different drift modes in the FT-2 plasma. To check this supposition and to identify modes the correlation technique [9] was used.
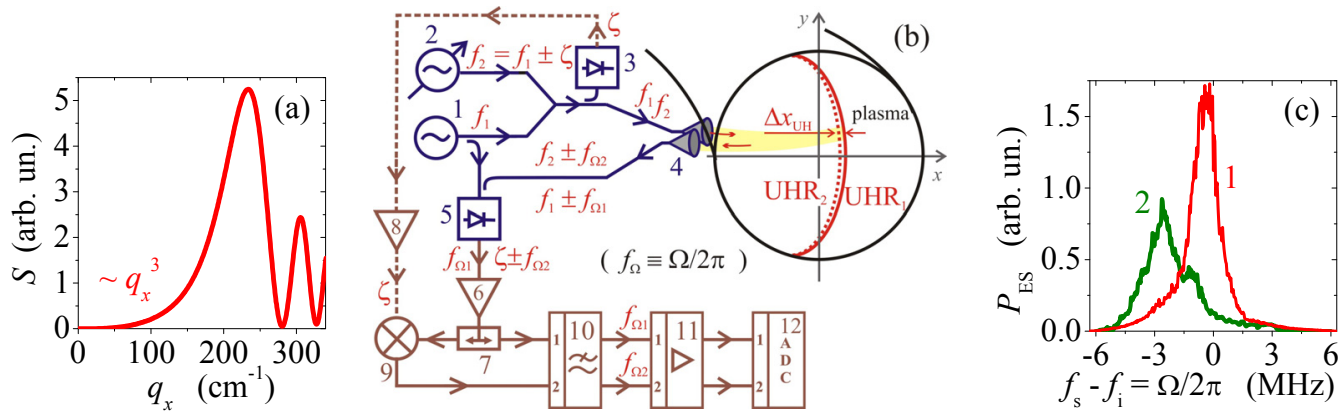

Fig. 1. (a) Dependence of the scattering efficiency on the fluctuation wave number. $(b)$ Asymmetric correlation scheme: 1 - microwave source (reference frequency $f_{1}$ and $20 \mathrm{~mW}$ output power); $2-$ microwave frequency tunable source (signal frequency $f_{2}, 20 \mathrm{~mW}$ power); 3, 5 - microwave mixers; 4 - $X$-mode vertically movable focusing double antenna set; 6,8 - low-noise amplifiers; 7 - directional coupler; 9 - RF-mixer; 10 - 7th-order low-pass filters with cutoff frequency $6 \mathrm{MHz} ; 11$ - lowfrequency amplifiers; $12-12$-bit $20 \mathrm{MHz}$ clock rate ADC. (c) ES frequency spectra: 1 - peripheral $\left(f_{\mathrm{i}}\right.$ $=61.9 \mathrm{GHz}) ; 2-$ in the gradient zone $\left(f_{\mathrm{i}}=65.8 \mathrm{GHz}\right)$.

Two signals at close probing frequencies with difference $\left|f_{2}-f_{1}\right|=\zeta=\{10,20, . .400\} \mathrm{MHz}$, corresponding to two slightly separated UHR layers in plasma, where the ES by fluctuations with low frequency $\Omega=2 \pi f_{\Omega}$ occurs, were measured simultaneously using the asymmetric correlation scheme [7], shown in Fig. 1(b). The microwave mixer 5 was fed by a local oscillator signal at the reference frequency $f_{1}$ in order to separate the frequency channels. Its homodyne converted $\left(f_{1} \pm f_{\Omega 1}-f_{1}= \pm f_{\Omega 1}\right)$ low-frequency output, corresponding to the scattered signal from the reference UHR position, was filtered in the first channel of the low-pass filter 10. The superheterodyne output of the mixer $5\left(f_{2} \pm f_{\Omega 2}-f_{1}=\zeta \pm f_{\Omega 2}\right)$, related to the signal from the second UHR layer, was supplied to the RF-mixer 9. There, it was homodyne converted to the low-frequency range using the local oscillator signal at the intermediate frequency $\zeta$, obtained on the microwave mixer 3. This signal was then filtered in the second channel of the low-pass filter 10. The phase tuning of the correlation scheme is performed at the intermediate frequency $\zeta$ by varying of the cable length between the microwave mixer 3 and RF-mixer 9, which is shown by dashed line in Fig. 1(b). After amplification and data acquisition two low-frequency signals were utilized for the cross-correlation function (CCF) computation. The normalized CCF, corresponding to the spectrum 2 in Fig. 1(c), obtained at the reference frequency $65.8 \mathrm{GHz}$ in $22 \mathrm{kA}$ discharge at $y_{\mathrm{a}}=1.5 \mathrm{~cm}$ is shown in Fig. $2(a)$. The $\Delta f_{\mathrm{i}}=f_{2}-f_{1}$ width of the high coherency region there decreases with growing turbulence frequency $\Omega$. As a result, the higher wave numbers $q_{r}$ should correspond to higher $\Omega$ in the cross-correlation spectrum (CCS), obtained by Fourier transform from the normalized CCF dependence on the UHR spatial separation $\Delta x_{\mathrm{UH}}=\zeta \partial x_{\mathrm{UH}} / \partial f_{\mathrm{i}}$ and multiplication by the ES signal frequency spectrum. The corresponding CCS, according to (11), proportional to the product of density fluctuations spectral power density and $S\left(q_{r}\right)$ is shown in Fig. 2(b). The imaginary part of the CCS, which should be zero in 
theory [6], determines the accuracy of our procedures. The turbulence wave number spectrum $|n|_{q_{r}}^{2}$ determined from this figure using (11) is shown in double logarithmic scale in Fig. 3(a). It has a pronounced maximum at $q_{r} \rho_{\mathrm{S}}=8$.
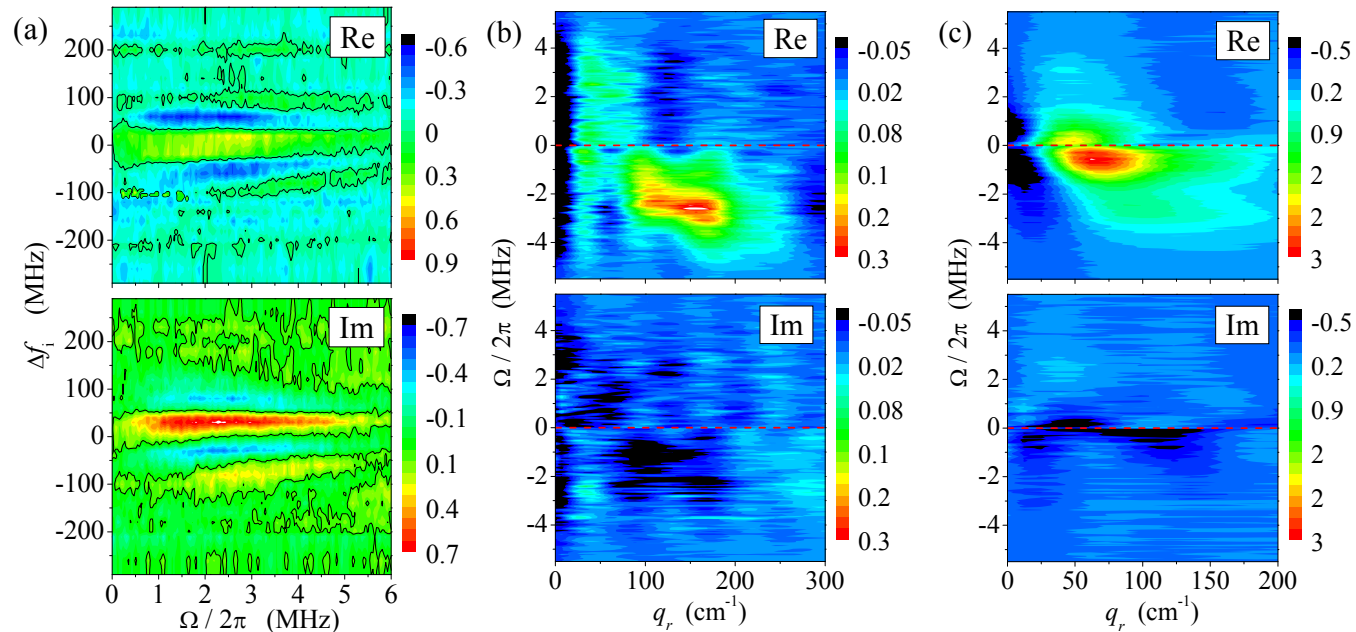

Fig. 2. (a) Normalized cross-correlation function (real and imaginary parts) in gradient zone where ETG is unstable $(65.8 \mathrm{GHz}, 22 \mathrm{kA})$. Black curves correspond to zero level. (b) Cross-correlation spectrum in gradient zone. (c) Cross-correlation spectrum in peripheral zone where ETG is still marginally unstable and LF-mode is dominating.
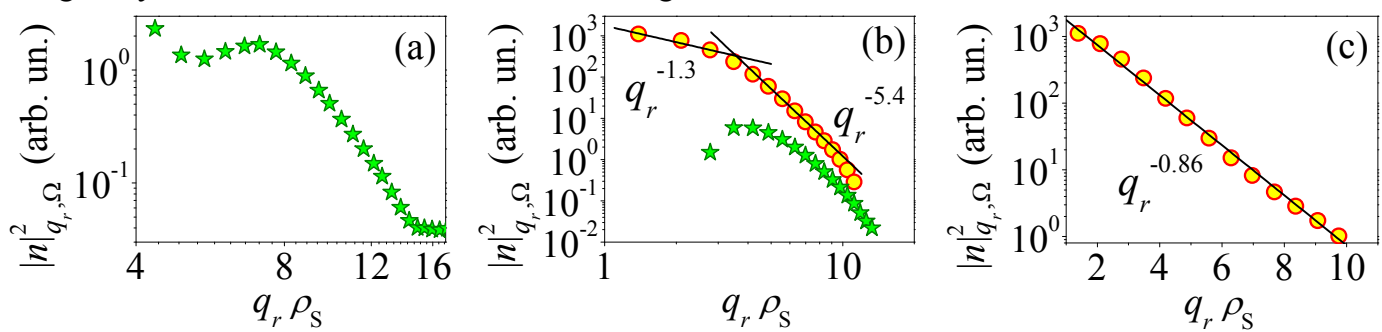

Fig. 3. (a) Turbulence wave number spectrum reconstruction for spectrum in gradient zone where ETG is unstable. (b) Turbulence wave number spectrum reconstruction for the two-component frequency spectrum. (c) The turbulence q-spectrum reconstruction for LF spectrum component.

The real part of the CCS spectrum $I_{q_{r}, \Omega}$ obtained in the typical peripheral FT-2 case when the condition $L_{T}<1.25 L_{n}$ is fulfilled but LF satellite is dominating in the ES power spectrum is shown in Fig. 2(c). It consists of two components very different in frequency. The LF satellite at $0.5 \mathrm{MHz}$ is larger there than the HF satellite at $2.4 \mathrm{MHz}$, which possesses higher $q_{r}$. The turbulence wave number spectrum $|n|_{q_{r}}^{2}$ determined from this figure using (11) is a result of superposition of two small-scale modes simultaneously excited in plasma at different frequencies. It is shown in double logarithmic scale in Fig. 3(b). It is knee-like for the LF mode with the rupture point at $q_{r} \rho_{\mathrm{S}}=3.5$ and scales approximately as $q_{r}^{-1.3 \pm 0.8}$ for $1.4<q_{r} \rho_{\mathrm{S}}<3.5$ and as $q_{r}^{-5.4 \pm 0.8}$ for $3.5<q_{r} \rho_{\mathrm{S}}<10$. When plotted in semi-logarithmic scale the spectrum represents a linear dependence on radial wave number, as shown in Fig. 3(c). These exponential spectra are typical for the outer radii where conditions for the ETG mode excitation are not satisfied [9]. This mode may be presumably identified with TEM mode. The HF mode radial wave number spectrum possesses a maximum at $q_{r} \rho_{\mathrm{S}}=4.2$. This wave number is comparable to that corresponding to the ETG instability growth rate maximum. The amplitude of the HF mode is 
sensitive to the violation of the ETG instability threshold condition [10] and therefore it may be attributed to the ETG mode.

\subsection{Plasma rotation measurements by the ES technique}

According to theoretical expectations [16], the phase velocity of drift waves responsible for $\mathrm{BS}$ is given by the following expression

$$
V_{\mathrm{ph}}=V_{\mathrm{dr}}\left(1+q_{\perp}^{2} \rho_{\mathrm{s}}^{2}\right)^{-1},
$$

where $q_{\perp}^{2}=q_{\theta}^{2}+q_{r}^{2}$ and $V_{\mathrm{dr}}$ is the drift wave phase velocity at $q_{\perp} \rho_{\mathrm{S}}<<1$, which is proportional to gradients of plasma parameters and depends on the drift mode type. According to (12) the phase velocity quickly decreases with growing wave number at $q_{\perp} \rho_{\mathrm{S}}>1$. Thus

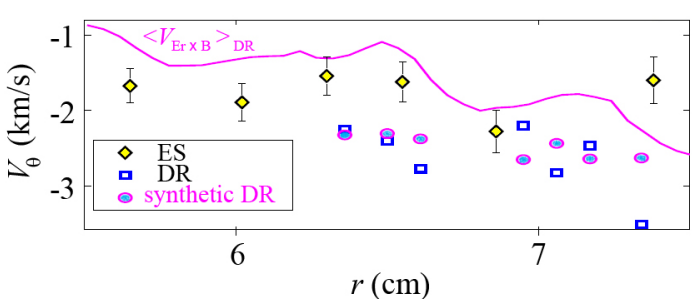

Fig. 4. The plasma and fluctuation rotation in FT2, as provided by GK modeling, ES, DR and synthetic DR.

it can be beneficial for the plasma poloidal velocity diagnostics to use the ES signal, which is produced by the small-scale turbulence component, possessing wave number in the range $q_{\perp} \rho_{\mathrm{S}} \gg 1$. To determine the rotation velocity profile from the dependence of the Doppler frequency shift $2 \pi f_{\mathrm{D}}=2 k_{\theta} V_{\theta}$ on the probing frequency, we need to know the electron density profile and, according to (1), to calculate the position of BS point and measure the radial wave number of fluctuations providing the largest contribution to the UHR BS signal with the correlation technique described above. The example of comparison of the velocity profile measured with ES, Doppler reflectometry (DR) and computed with Elmfire gyrokinetic (GK) code [12] is shown in Fig. 4. As it is seen in the figure, the GK modeling utilizing the synthetic diagnostics correctly describes the velocity profile of fluctuations contributing to the DR signal, however this velocity is different from the plasma velocity due to contribution of the phase velocity. On contrary, in the case of ES diagnostics the measured fluctuation velocity is close to computed plasma velocity.

\subsection{GAM investigation by ES diagnostics}

The method of poloidal rotation dynamics study with ES is, in essence, similar to that proposed in [13] for Doppler reflectometry. The quadrature (i.e. with IQ detection) ES scheme was used for this purpose in the experiments. The moveable focusing double $X$-mode antennae set at the high field side was positioned vertically at $y=+15 \mathrm{~mm}$ above the equatorial plane for Doppler ES measurements. The pair of sine-cosine (IQ) homodyne signals at frequencies of the turbulent fluctuations was used for reconstruction of the bilateral spectrum equivalent to the heterodyne ES spectrum. An example of the ES spectrum averaged over $1.6 \mathrm{~ms}$ of hydrogen $19 \mathrm{kA}$ discharge is shown in Fig. 5(a) by the red curve, where $f$ corresponds to the turbulence frequency. The spectrum is wide and has a significant Doppler frequency shift $f_{\mathrm{D}} \sim 800 \mathrm{kHz}$ in the electron diamagnetic drift direction. However when calculated without averaging in $3.2 \mu \mathrm{s}$ window the power spectrum $\left(P_{\mathrm{ES}}\right)$ appears to be narrow and possesses a frequency shift different from the averaged one given by curve 1 (see blue curve 2 in Fig. 5(a)). The variation of the non averaged spectrum during $1.6 \mathrm{~ms}$ finaly results in the red curve. The Doppler frequency $\operatorname{shift} f_{\mathrm{D}}$ behaviour estimated from the temporal variation of the non-averaged spectra as 


$$
f_{\mathrm{D}}(t)=\int f\left|P_{\mathrm{ES}}(f, t)\right| d f\left(\int \mid P_{\mathrm{ES}}(f, t) d f\right)^{-1}
$$

is presented in Fig. 5(b) by the red curve, whereas the mean values determined in $1.6 \mathrm{~ms}$ windows are shown by open circles. The spectrum of the $f_{\mathrm{D}}(t)$ dependence, calculated with
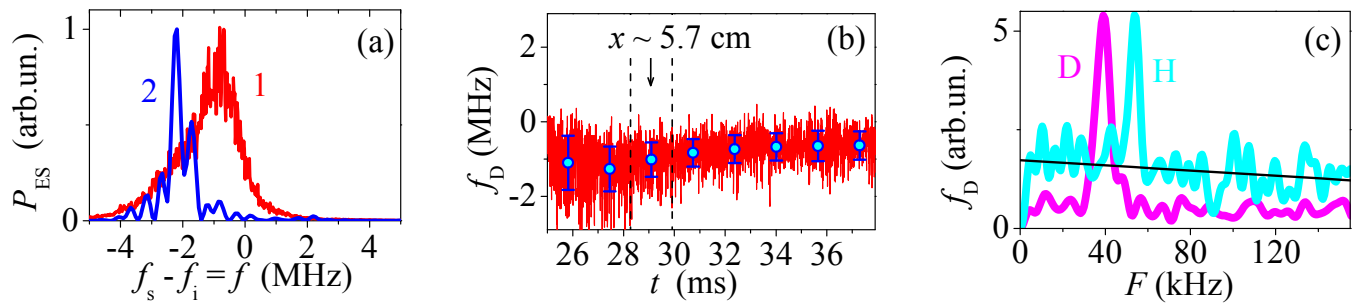

Fig. 5. (a) ES spectra measured in different temporal intervals $(1-63$ averages within $1.6 \mathrm{~ms} ; 2-$ $3.2 \mu \mathrm{s}$ w/o averaging). (b) Temporal behavior of ES Doppler frequency shift. (c) The spectrum of the $f_{\mathrm{D}}(t)$ dependence.

averaging over 8 samples (with FFT windows taken one by one) within an $1.6 \mathrm{~ms}$ interval between the dashed lines in Fig. 5(b) is presented in Fig. 5(c) by cyan curve. An intensive spectral line is clearly seen in the spectrum at frequency $F \sim 54 \pm 1.2 \mathrm{kHz}$ close to the value theoretically predicted for GAM. The black solid line there corresponds to the mean noise level, which can be attributed to perturbations of radial electric field $E_{r}$ and density, as well as distortions of the UHR and magnetic surfaces. The ratio of the line's amplitude $P_{\max } \sim 5.41 \pm 0.03$ to the noise level $P_{\mathrm{n}} \sim 1.55 \pm 0.47$ at peak's frequency is a contrast $C=P_{\max } / P_{\mathrm{n}} \sim 3.5 \pm 1.1$. The magenta curve in Fig. 5(c) corresponds to the $f_{\mathrm{D}}(t)$ spectrum obtained in the $19 \mathrm{kA}$ deuterium discharge. As it is seen in the figure an intensive line persists in this spectrum as well. The contrast of the line is higher than in hydrogen whereas the frequency is smaller, which is natural for the GAM. The spatial scan of the $f_{\mathrm{D}}(t)$ spectrum yields the radial dependence of the line contrast and frequency of the shown in Fig. $6(a, b)$ by circles. The mean value of the $f_{\mathrm{D}}(F)$ noise in the terms of contrast corresponds to the level $C=1$. The grey curve in Fig. $6(a)$ is the rms value of this noise. The theoretical estimations of the GAM frequency $\left(F_{\mathrm{G}}\right)$ accounting for spatial variation of plasma parameters are also plotted in Fig. $6(b)$ by blue and red solid lines for hydrogen and deuterium accordingly. These estimations are based on the analytical prediction of the GAM frequency, obtained in [13] for plasmas with two ion species (the main component and the $\mathrm{O}^{8+}$ impurity component). The agreement of theory and measurements permits the conclusion that the dominant line in the $f_{\mathrm{D}}(F)$ spectrum results from the interaction with GAMs. It should be mentioned that the spatial distribution of the GAM frequency obtained from $E_{r}(t)$ dependence in ELMFIRE simulation $[12,15]$ was also very close to the experimental dependence in Fig. $6(a, b)$.

In order to investigate the GAM radial structure two ES signals at probing frequencies $f_{1}$ and $f_{2}$, corresponding to UHR spatial separation $\Delta L$ in the plasma, are measured simultaneously by two homodyne channels. In general, the peculiarity of the homodyne spectrum is an overlapping of the spectral components with the same turbulence frequencies but with opposite phase velocities. Because of the large Doppler frequency shift of the quadrature spectrum (see Fig. 5(a)) the overlapping effect is, however, small enough to ensure reliable determination of the variable Doppler frequency shift based on homodyne spectra. Therefore, using the homodyne spectra two oscillating signals $f_{\mathrm{D} 1}(t)$ and $f_{\mathrm{D} 2}(t)$ can be reconstructed in the same manner as it was described for the quadrature spectrum (see formula 13). Due to the modulation 
of both signals a high level of correlation between them can be expected just at the GAM frequency. The coherency of two signals is shown in Fig. 6(c) as a function of frequency and spatial separation of measurement points whereas the cross-phase behavior is shown in Fig. 6(d) in comparison to the GK modeling predictions [15]. As a result of correlative measurements we obtain the GAM correlation length of $0.7 \mathrm{~cm}$ and GAM wavelength of $2.4 \mathrm{~cm}$.
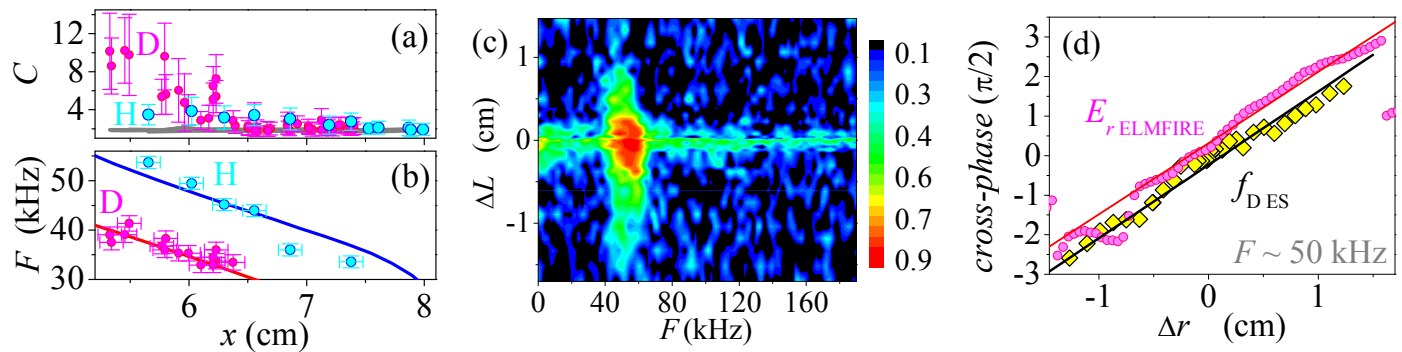

Fig. 6. GAM (a) contrast and (b) frequency spatial distributions. (c) Radial coherency of plasma oscillations only meaningful at the GAM frequency. (d) The cross-phase spatial behavior at the GAM frequency.

\section{Investigation of anomalous absorption in the helicon discharge}

A substantial limitation for application of the ES scheme in plasma experiments is due to the UHR layer accessibility condition. In toroidal devices possessing inhomogeneous magnetic field this condition prescribes that the probing waves have to be launched from the high magnetic field side of the torus. As a consequence, the maximum density for operation is limited by the condition $\omega_{p e}^{2}<\left(\omega_{c e}+\omega_{i}\right) \omega_{i}$. In this approach, the probing wave enters the UHR via the cyclotron resonance layer and, hence, suffers from absorption which can be strong in large fusion devices. In spite of the mentioned density limitation the ES technique can be used in the opposite situation of a very high density $\left(\omega_{\mathrm{pe}}>>\omega_{\mathrm{ce}}\right)$, which is typical for helicon discharge or spherical tokamak. Although the probing wave cannot enter the UHR across the central plasma region, the resonance can be reached (from the plasma edge in helicon plasma and from the low magnetic field side in spherical tokamak) through the thin evanescent region between the cutoff and the UHR. The feasibility of the ES diagnostic has been demonstrated for the high-density plasma conditions of a helicon discharge in [16], where relatively high level of ion sound turbulence presumably produced by parametric decay of the helicon pump wave into short ionacoustic and Trivelpiece-Gould waves was detected [17]. The dispersion of the decay waves was determined in the CES experiments utilizing $9 \mathrm{GHz}$ and $28 \mathrm{GHz}$ plasma probing across the evanescent layer [18]. In the latter case the ES signal normalized CCF real and imaginary parts are shown in Fig. 7(a). The Fourier transform of this CCF results in 2D spectrum, shown in Fig. 7(b). This spectrum demonstrates excitation of the low-frequency mode possessing the
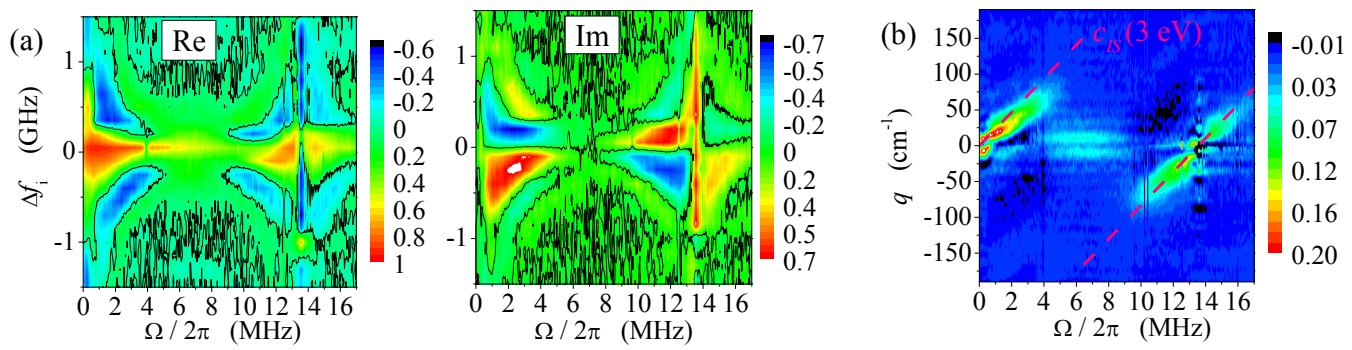

Fig. 7. (a) The CCF real and imaginary parts in the low pressure helicon discharge ES experiment. (b) The real part of the ES signal 2D spectrum. 
linear dispersion relation with the phase velocity equal to the acoustic one at electron temperature of $3 \mathrm{eV}$ and a complementary spectral component down-shifted from the helicon wave frequency $13.56 \mathrm{MHz}$. This component is presumably the electron plasma wave, which, as it is seen from Fig. 7(b) satisfies the decay condition for the helicon decay into the ion acoustic and electron plasma waves.

\section{Conclusions}

Summarizing the results of correlative enhanced scattering diagnostics development during the last decade we should like to point out to the clear demonstration of its potential for studying of the small-scale waves or fluctuations in fusion and low-temperature plasmas. With CES the first observations of the ETG mode in tokamak plasma, as well as of anomalous mechanism of the helicon discharge formation were made. It was shown that the CES allows reliable measurements of plasma rotation velocity and its dynamics as well as the spatial structure of GAMs. It was demonstrated that the excellent spatial resolution of this technique provides sufficient conditions for benchmarking of the anomalous transport gyrokinetic codes.

\section{References}

[1] K.M. Novik and A.D. Piliya, Plasma Phys. Control. Fusion 35 (1994) 357.

[2] A.D. Gurchenko, E.Z. Gusakov, V.L. Selenin et al., Physics of Plasmas 8 (2001) 2224.

[3] E.Z. Gusakov and A.D. Piliya, Sov. Tech. Phys. Lett. 18 (1992) 325.

[4] V.I. Arkhipenko et al., Plasma Phys. Control. Fusion 37A (1995) 347.

[5] V.I. Arkhipenko, V.N. Budnikov, E.Z. Gusakov et al., Tech. Phys. Lett. 19 (1993) 20.

[6] E.Z. Gusakov et al., Plasma Phys. Control. Fusion 42 (2000) 1033.

[7] E.Z. Gusakov et al., Plasma Phys. Control. Fusion 48 (2006) A371.

[8] E.Z. Gusakov, A.D. Gurchenko, A.B. Altukhov et al. Plasma Phys. Control. Fusion 48 (2006) B443.

[9] A.D. Gurchenko et al., Plasma Phys. Control. Fusion 52 (2010) 035010.

[10] A.D. Gurchenko, E.Z. Gusakov, S.I. Lashkul et al., Plasma Physics Reports 39 (2013) 337.

[11] A.D. Gurchenko and E.Z. Gusakov, Plasma Phys. Control. Fusion 52 (2010) 124035.

[12] S. Leerink, V.V. Bulanin, A.D. Gurchenko et al., PRL 109 (2012) 165001.

[13] G.D. Conway et al., Plasma Phys. Control. Fusion 47 (2005) 1165.

[14] W. Guo, S. Wang and J. Li, Phys. Plasmas 17 (2010) 112510.

[15] E.Z. Gusakov, A.B. Altukhov, V.V. Bulanin et al., Plasma Phys. Control. Fusion 55 (2013) 124034.

[16] N.M. Kaganskaya, M. Krämer and V. L. Selenin, Phys. Plasmas 38 (2001) 4308.

[17] A.B. Altukhov, E.Z. Gusakov, M.A. Irzak et al., Phys. Plasmas 12 (2005) 022310.

[18] M. Kramer, Yu.M. Aliev, A.B. Altukhov et al., Plasma Phys. Control. Fusion 49 (2007) A167. 